\section{PERFIL E ANÁLISE DE PARETO DOS ERROS DE DISPENSAÇÃO DE UM HOSPITAL PÚBLICO}

Rafaela Sirtoli ${ }^{1}$

Eliz Cassieli Pereira Pinto ${ }^{1}$

Carine Fieira ${ }^{1}$

Jeferson Giovan Volkweis ${ }^{1}$

Suelem Tavares da Silva

Penteado ${ }^{1}$

Luciane de Fátima Caldeira ${ }^{1}$

Introdução: Estratégias de avaliação e monitoramento de processos subsidiam o enfrentamento de erros de medicação (EM), os quais constituem parte majoritária dos eventos adversos (EA) em pacientes hospitalares. Muitos estudos dizem respeito aos erros de prescrição e administração, porém poucos discutem os impactos dos erros de dispensação. Objetivo: Descrever o perfil dos erros de dispensação e quantificar as discrepâncias geradas pelo indicador, em um hospital de alta complexidade. Métodos: Estudo transversal e retrospectivo dos erros de dispensação evitados pelo farmacêutico. Uma vez identificadas, as discrepâncias foram classificadas e contabilizadas, gerando um indicador do processo de trabalho (número de erros evitados/número de tiras dispensadas x 100). Posteriormente, fora realizada uma Análise de Pareto, a fim de priorizar ações de resolução de problemas. Resultados: Obteve-se um total de 27.980 prescrições, as quais originaram 48.451 tiras de medicamentos. Os erros de dispensação encontrados somaram 1.453. O índice de erros de dispensação foi de 3 erros a cada 100 tiras. Os erros que ocorreram com maior frequência foram falta de medicamento prescrito e troca de horário do medicamento, representando juntos $61 \%$ da totalidade dos erros. Os mesmos erros foram identificados na análise no Diagrama de Pareto como os principais alvos de intervenção na melhoria dos processos. Conclusões: A ocorrência de erros encontrada através do indicador "Índice de Erros de Dispensação" foi considerada baixa, frente aos dados encontrados na literatura. Através da Análise de Pareto, foi possível identificar os erros mais frequentes, para posterior planejamento de ações de melhoria, com vistas ao aumento da segurança do paciente.

Palavras-chave: Erros de medicação; segurança do paciente; indicadores de qualidade em saúde.

\section{INTRODUÇÃO}

O Programa Nacional de Segurança do Paciente (PNSP) foi instituído no Brasil em 2013, com o objetivo de promover práticas seguras em estabelecimentos de saúde. Através da instituição de protocolos, esse visa garantir a segurança do paciente inserindo novas estratégias para o aperfeiçoamento da assistência à saúde ${ }^{1}$.

Dentre os protocolos estabelecidos pelo PNSP, está o "Protocolo de Segurança na Prescrição e de Uso e Administração de Medicamentos”, com a finalidade de estabelecer medidas que minimizem a ocorrência de erros de medicação em todas as etapas da cadeia terapêutica ${ }^{2}$. Os erros de medicação (EM) constituem parte majoritária dos eventos adversos (EA) em pacientes hospitalares ${ }^{3}$.Uma revisão sistemática publicada em 2008 evidenciou a magnitude dos erros associados à medicação no contexto dos EA, constituindo o segundo tipo mais prevalente dentre os eventos elencados pela pesquisa 4. Além dos potenciais danos causados ao indivíduo, devem ser considerados também os custos adicionais do tratamento, resultado de um aumento do tempo de internação e possíveis complicações ${ }^{5}$.

Nesse sentido, a instituição de estratégias de avaliação e monitoramento de processos subsidiam o enfrentamento aos potenciais erros relacionados a medicamentos 6,7. Dentre as ferramentas recomendadas pelo Ministério da Saúde (MS), estão os indicadores em saúde, definidos como dados ou informações numéricas que quantificam, entre outras varáveis, o desempenho de processos e da organização como um todo ${ }^{8}$.
Os EM podem ser definidos como qualquer evento evitável que, de fato ou potencialmente, pode levar ao uso inadequado de medicamento. Esse conceito implica que o uso inadequado pode ou não lesar o paciente. Tais eventos podem estar relacionados a diversos estágios da assistência à saúde, entre elas prescrição, dispensação, administração ${ }^{9-10}$.

Contudo, a maioria das pesquisas diz respeito aos erros de prescrição e administração ${ }^{11}$, enquanto poucos estudos são publicados sobre os erros de dispensação no Brasil e no mundo.

Ademais, torna-se relevante identificar a categoria de EM, bem como seus determinantes, a fim de direcionar estratégias de enfrentamento para a prevenção dos mesmos ${ }^{2}$.

O objetivo do presente trabalho fora descrever o perfil dos erros de dispensação em uma farmácia de um hospital terciário do Estado do Paraná, através da quantificação e classificação dos mesmos pelo indicador de taxas de erros na conferência de medicamentos antes da dispensação, e, através da Análise de Pareto, subsidiar ações de gestão para melhoria de processos.

\section{MÉTODOS}

Trata-se de um estudo transversal e retrospectivo dos erros de dispensação que foram evitados através da instituição da conferência de tiras de medicamentos pelo farmacêutico hospitalar.

O estudose desenvolveu em um Hospital Público
1 Universidade Estadual do Oeste do Paraná

Recebido: $27 / 02 / 2018$

Revisado: 13/06/2018

Aceito: 25/06/2018

Como citar este artigo: Sirtoli R, Pinto ECP, Fieira C, Volkweis JG, Penteado STS e Caldeira LFC. Perfil e análise de pareto dos erros de dispensação de um hospital público. Rev.

Bras. Farm. Hosp. Serv. Saúde, 9(2): pag-pag, 2019.

Doi: 10.30968/rbfhss.2018.092.007
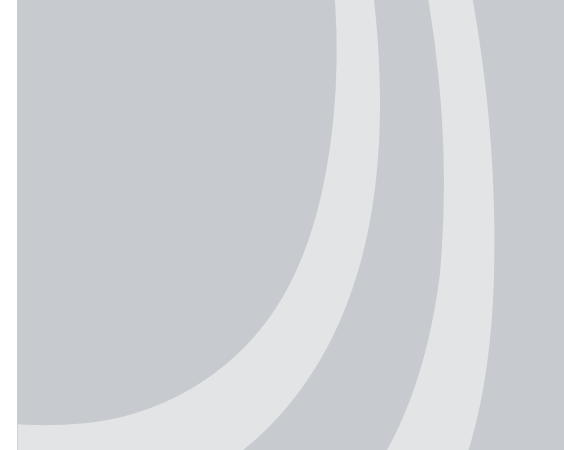

Autor correspondente:

Rafaela Sirtoli Universidade Estadual do Oeste do Paraná, Av. Tancredo Neves, 3224 - Santo Onofre, Cascavel PR, Brasil. CEP: 85806-470 rafaela.sirtoli@hotmail.com 
do Oeste do Paraná de nível terciário, com atendimento unicamente SUS e capacidade para 195 leitos, financiado pelo Governo do Estado e Federal.

O Serviço de Farmácia do presente estudo registra como erros de dispensação os desvios que ocorrem durante a separação, baixa eletrônica, confecção de tiras e dispensação dos medicamentos.

O serviço farmacêutico da presente instituição tem início com a análise das prescrições, seguida da separação dos medicamentos prescritos, baixa eletrônica e confecção das tiras. O sistema de distribuição é do tipo individualizado, no qual são fornecidas tiras por período, para cada paciente. As prescrições são válidas para 24 horas, gerando assim três tiras: manhã, tarde e noite, sendo essas confeccionadas concomitantemente.

A conferência de tiras foi implantada no serviço de farmácia do hospital no ano de 2012. Antes da dispensação à equipe da Enfermagem, são conferidas pelo farmacêutico as tiras dos turnos manhã e noite dos seguintes setores de internação: Clínica Médica e Cirúrgica; Maternidade; Pediatria; Unidade de Terapia Intensiva Adulto (UTI); e Ortopedia e Neurologia.

As tiras dispensadas no turno da tarde não foram contabilizadas no presente estudo, visto que, devido à logística do serviço, as mesmas não passam pela conferência do farmacêutico.

Durante o processo de conferência, o farmacêutico avalia possíveis discrepâncias entre a tira, previamente confeccionada pelo serviço de farmácia hospitalar, e a prescrição correspondente. Uma vez identificadas, as discrepâncias são classificadas quanto à sua categoria, a fim de gerar um indicador do processo de trabalho. Visto que a conferência foi implementada como uma barreira adicional aos erros de medicação, as inconsistências constatadas são encaminhadas à correção e posteriormente dispensadas.

O Indicador do presente estudo foi denominado "Índice de Erros de Dispensação”. O numerador da fórmula do indicador consiste no número total de erros identificados durante a conferência, enquanto o denominador é representado pelo número total de tiras confeccionadas. $\mathrm{O}$ resultado obtido é expresso na potência de dez, gerando um índice Erro/ Tiras.

A metodologia de categorização dos erros foi baseada na classificação segundo a The National Coordinating Council for Medication Error Reporting and Prevention (NCCMERP) sendo adaptada à realidade local. Com base nessa, foram considerados erros de dispensação: falta de identificação do paciente; troca de medicamento; falta de medicamento; erro de dose sobredose ou subdose; troca de horário e troca de forma farmacêutica.

Quanto à técnica estatística utilizada, aplicou-se o Diagrama de Pareto, a fim de nortear a tomada de decisão. Essa permite selecionar e priorizar um número pequeno de fatores que tem grande impacto na melhoria dos processos. O Princípio de Pareto baseia-se na ideia de que $80 \%$ dos resultados correspondem a apenas $20 \%$ dos fatores, justificando a priorização.

Foram analisados os dados originados entre o período de 01 de janeiro a 31 de dezembro de 2015. A tabulação e análise estatística foram realizadas no Software Microsoft Excel ${ }^{\circledR} 2013$. Os erros foram classificados quanto à sua categoria e avaliados quanto à distribuição da frequência. Posteriormente, fora realizada uma Análise de Pareto, a fim de priorizar ações para a resolução de problemas. $\mathrm{O}$ estudo foi aprovado pelo Comitê de Ética em Pesquisa (CEP), sob parecer no 1.872.685.

\section{RESULTADOS E DISCUSSÃO}

Obteve-se um total de 27.980 prescrições, as quais originaram 48.451 tiras de medicamentos, no período estudado. Os erros de dispensação encontrados durante a conferência das tiras pelo farmacêutico somaram 1.453. O índice de erros de dispensação foi de 3 erros a cada 100 tiras.

Para a discussão, é importante considerar que, embora a pesquisa sobre os erros de dispensação no Brasil venha ganhando espaço nos últimos anos, a literatura a respeito do tema ainda é escassa. Assim, a comparação entre os resultados deve ser cautelosa, visto que há grande divergência nas metodologias aplicadas e na expressão dos resultados. Ademais, vale ressaltar a diferença entre os cenários e serviços farmacêuticos já estudados, a qual complexifica ainda mais o contexto de comparação.

Até o presente momento não foram identificados trabalhos que empregassem metodologia semelhante à do presente estudo (indicador expresso em índice), uma vez que a construção do indicador foi baseada na realidade local - onde é calculado o número de erros por tira, e não número de erros por medicamentos dispensados - inviabilizando sua expressão em taxa ${ }^{7,12,13}$.

Apesar de não poderem ser comparados diretamente, em uma conversão hipotética, a taxa encontrada (3\%) seria demasiadamente inferior aos relatos da literatura, considerando que o denominador aumentaria exponencialmente se o número de medicamentos contidos em cada tira fosse considerado em detrimento do denominador utilizado - erro por uma unidade de tira.

Tal resultado pode estar relacionado ao sistema de separação empregado na farmácia estudada. Acredita-se que o fato de cada etapa do processo (separação dos medicamentos prescritos, baixa eletrônica e confecção das tiras) ser realizada por um colaborador diferente pode constituir uma barreira adicional para a ocorrência de erros anteriormente à conferência farmacêutica.

A classificação dos erros está disposta na Tabela 1. Os erros que ocorreram com maior frequência foram "falta de medicamento" e "troca de horário do medicamento", representando juntos $61 \%$ da totalidade dos erros. É digno de nota que a denominação "falta de medicamento" se refere à falta do mesmo na tira conferida, e não falta do medicamento na Instituição.

Tabela 1. Classificação e incidência dos erros de dispensação por setor de internação.

\begin{tabular}{|c|c|c|c|c|c|c|c|}
\hline $\begin{array}{l}\text { Classificação } \\
\text { dos erros }\end{array}$ & $\begin{array}{c}\text { UTI } \\
\text { Adulto }\end{array}$ & $\begin{array}{l}\text { Clínica } \\
\text { Médica e } \\
\text { Cirúrgica }\end{array}$ & $\begin{array}{c}\text { Ortopedia/ } \\
\text { Neurologia }\end{array}$ & Pediatria & Maternidade & Total & $\%$ \\
\hline $\begin{array}{l}\text { Falta de } \\
\text { medicamento }\end{array}$ & 54 & 146 & 102 & 59 & 88 & 449 & $31 \%$ \\
\hline $\begin{array}{l}\text { Troca de } \\
\text { horário do } \\
\text { medicamento }\end{array}$ & 40 & 181 & 112 & 55 & 51 & 439 & $30 \%$ \\
\hline $\begin{array}{l}\text { Erro de dose - } \\
\text { Subdose }\end{array}$ & 30 & 61 & 41 & 33 & 21 & 186 & $13 \%$ \\
\hline $\begin{array}{l}\text { Erro de dose - } \\
\text { Sobredose }\end{array}$ & 26 & 44 & 30 & 32 & 21 & 153 & $11 \%$ \\
\hline $\begin{array}{l}\text { Troca de } \\
\text { medicamento } \\
\text { no pacote }\end{array}$ & 8 & 39 & 46 & 22 & 13 & 128 & $9 \%$ \\
\hline $\begin{array}{l}\text { Troca } \\
\text { da forma } \\
\text { farmacêutica }\end{array}$ & 7 & 24 & 17 & 7 & 15 & 70 & $5 \%$ \\
\hline $\begin{array}{l}\text { Falta de } \\
\text { identificaçāo }\end{array}$ & 3 & 8 & 9 & 4 & 4 & 28 & $2 \%$ \\
\hline $\begin{array}{l}\text { Troca de } \\
\text { pacote no } \\
\text { horário }\end{array}$ & 0 & 0 & 0 & 0 & 0 & 0 & $0 \%$ \\
\hline TOTAL & 168 & 503 & 357 & 212 & 213 & 1453 & \\
\hline$\%$ & 11 & 35 & 25 & 14 & 15 & 100 & \\
\hline
\end{tabular}

Estudo conduzido por Galvão et al. (2012), corrobora com os resultados encontrados, no qual "a falta de medicamento" foi o principal erro constatado na dispensação (41,66\%). Taxa semelhante foi descrita na pesquisa de Albuquerque et al. (2016) onde a omissão de doses representou $35,93 \%$ dos erros. Tais achados sinalizam para uma fragilidade na etapa de separação dos medicamentos prescritos, indicando necessidade de revisão dessa rotina ${ }^{12,14}$

Por sua vez, o segundo erro mais frequente - troca de horário do medicamento $(30 \%)$-, reflete uma deficiência na etapa final do processo, visto que é somente no momento da confecção das tiras que 
os medicamentos são segregados por turno, favorecendo a ocorrência de tal falha no processo. Assim, pode-se explicar a disparidade dos resultados com os encontrados na literatura, onde esse tipo de erro não é tão prevalente. Em estudos onde os medicamentos são separados por horário de administração, as taxas encontradas foram cerca de duas vezes inferiores às descritas acima ${ }^{13,14}$.

Uma vez aplicada a Análise de Pareto, reiterou-se os erros falta de medicamento e troca de horário do medicamento como principais alvos de intervenção (Figura 1).

Apesar de menos frequentes, os erros de dose - sub ou sobredose também contribuem para a totalidade dos $80 \%$, conforme Princípio de Pareto. Contudo, se houver resolubilidade dos dois fatores majoritários, mais da metade dos erros serão prevenidos.

Figura 1 - Análise de Pareto quanto à incidência de erros de dispensação.

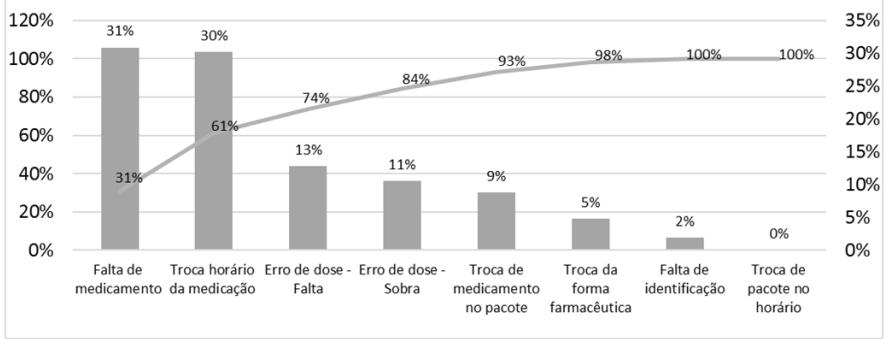

Assim, a Análise de Pareto se mostrou uma ferramenta de gestão útil e prática no direcionamento de ações para melhoria dos processos de dispensação.

\section{CONCLUSÕES}

Observamos que a ocorrência de erros de dispensação encontrada através do indicador Índice de Erros de Dispensação foi baixa. Através da Análise de Pareto, foi possível verificar que os quatro erros mais frequentes correspondem a mais de $80 \%$ dos erros de dispensação encontrados, sendo eles: Falta de medicamento; Troca de horário de medicamento; Erro de dose - subdose; e Erro de dose - sobredose. Assim, esses devem ser priorizados no planejamento de melhorias no processo a fim de evitar a ocorrência dos mesmos.

Ainda, indicadores em saúde - semelhantes ao utilizado nesse estudo - são instrumentos importantes de avaliação de processos. Entretanto, vale ressaltar que, esses são ferramentas de direcionamento, necessitando da implantação conjunta de diferentes estratégias de enfrentamento, como as barreiras empregadas no serviço de farmácia hospitalar estudado.

Por fim, erros de medicação, enquanto eventos evitáveis, são passíveis de intervenções. Nesse sentido, os resultados do presente estudo podem subsidiar o planejamento de ações de melhoria, tendo em vista uma maior segurança do paciente.

\section{Fontes de Financiamento}

A pesquisa não recebeu financiamento para a sua realização.

\section{Conflito de Interesses}

Os autores declaram inexistência de conflitos de interesses.

\section{Colaboradores}

RS,ECPP, CF e JGV: Concepção e planejamento do projeto em pesquisa; análise e interpretação dos dados; redação do artigo; aprovação final da versão a ser publicada; responsabilidade por todas as informações do trabalho, garantindo exatidão e integridade de qualquer parte da obra. STSP e LFC: Concepção e planejamento do projeto em pesquisa; revisão crítica relevante do conteúdo intelectual; aprovação final da versão a ser publicada; responsabilidade por todas as informações do trabalho, garantindo exatidão e integridade de qualquer parte da obra.

\section{REFERÊNCIAS}

1. Brasil. Ministério da Saúde. Portaria no 529, de 1o de abril de 2013. Institui o Programa Nacional de Segurança do Paciente (PNSP). Diário Oficial da União, Brasília, DF, 2 abr. 2013. Seção 1, p. 43.) Disponível em: < http://www.cvs.saude.sp.gov.br/up/U_PT-MSGM-529_010413.pdf> Acesso em: 30 nov. 2016.

2. Brasil. Ministério da Saúde. Agência Nacional de Vigilância Sanitátia. Fundação Oswaldo Cruz. Fundação Hospitalar do Estado de Minas Gerais. Protocolo de segurança na prescrição, uso e administração de medicamentos, 2013. Disponível em: <http://www.hospitalsantalucinda.com. br/downloads/prot_meficamentos.pdf> Acesso em: 30 nov. 2016.

3. Bates DW, Boyle DL, Vander Vliet MB et al. Relationship between medication errors and adverse drug events. J Gen Intern Med, 1995,10(4):199-205.

4. De Vries EN, Ramrattan MA, Smorenburg SM et al. The incidence and nature of in-hospital adverse events: a systematic review. Quality \& Safety in Health Care, 2008, 17(3):216-223.

5. Silva AEBC, Cassiani SHDB. Análise prospectiva de risco do processo de administração de medicamentos anti-infecciosos. Rev Lat Am Enfermagem, 2013, 21:233-41

6. Langebrake C, Hilgarth H. Clinical pharmacists' interventions in a German University Hospital, Pharm World Sci, 2010, 32:194-199.

7. Instituto para Práticas Seguras no Uso de Medicamentos. Programa nacional de Segurança do Paciente: Indicadores para Avaliação da Prescrição, do Uso e da Administração de Medicamentos - Parte I. Boletim Informativo ISMP Brasil, maio 2016, v. 5, n. 1.

8. Fundação Prêmio Nacional da Qualidade. Rumo à excelência: critérios para avaliação do desempenho e diagnóstico organizacional. São Paulo: Fundação Nacional da Qualidade, 2005.

9. National Coordinating Council for Medication Error Reporting and Prevention. Taxonomy of medication errors - 1998-1999 [online]. Disponível em: <http://www.nccmerp.org/about-medication-errors $>$ Acesso em: 12 jun. 2018.

10. American Society of Healthy-System Pharmacists. Suggested definitions and relationships among medication misadventures, medication errors, adverse drug events, and adverse drug reactions. Am J Health Syst Pharm, 1998, 55(2):165-6.

11. James KL, Barlow D, Mcartney R et al. Incidence, type and causes of dispensing errors: a review of the literature. 2009;9-30.

12. Albuquerque PMS, Dantas JG, Vasconcelos LA et al. Identificação de erros na dispensação de medicamentos em um Hospital Oncológico. Rev. Bras. Farm. Hosp. Serv. Saúde, 2012, 3(1):15-18.

13. Rissato MAR. Erros de dispensação de medicamentos em hospital universitário no Paraná [tese]. São Paulo: Faculdade de Saúde Pública; 2012 Disponível em <http://www.teses.usp.br/teses/disponiveis/6/6135/ tde-17052012-120257/pt-br.php> Acesso em: 20 dez 2016.

14. Galvão AL, Oliveira AM, Carvalho FB et al. Identificação e distribuição dos erros de dispensação em uma farmácia hospitalar: um estudo comparativo no município de Salvador Bahia. Rev. Ci.Med.Biol, 2012, 11(2):201-206. 